\title{
Effect of Concomitant Administration of Trospium Chloride Extended Release on the Steady-State Pharmacokinetics of Metformin in Healthy Adults
}

\author{
Michael G. Oefelein • Warren Tong • \\ Sam Kerr · Kavitha Bhasi • Rina K. Patel • \\ Dale Yu
}

Published online: 17 January 2013

(c) The Author(s) 2013. This article is published with open access at Springerlink.com

\begin{abstract}
Background Overactive bladder (OAB) is often associated with a number of co-morbid medical conditions, including diabetes mellitus. This may necessitate several concomitant treatments, thus creating the potential for drug-drug interactions (DDIs). Trospium is renally eliminated, not metabolized via cytochrome P450; therefore, cytochrome P450 DDIs are unlikely. However, coadministration with another renally eliminated drug (e.g., metformin) may theoretically result in a DDI.

Objective The objective of this study was to evaluate the pharmacokinetics (plasma and urine) and safety/tolerability of the coadministration of trospium chloride extended release (XR) and metformin under steady-state conditions in healthy male and female subjects.

Methods In a single-centre, randomized, open-label, twogroup, two-period study in healthy males and females aged $18-45$ years, 44 subjects received oral metformin $500 \mathrm{mg}$ twice daily for 3.5 days during one period, and oral trospium chloride XR $60 \mathrm{mg}$ once daily for 10 days, followed by trospium chloride XR $60 \mathrm{mg}$ once daily for 4 days plus metformin $500 \mathrm{mg}$ twice daily for 3.5 days during the other period. The two periods occurred in a crossover fashion, separated by a 3-day washout period.

Results Trospium chloride XR coadministration did not alter metformin steady-state pharmacokinetics. Metformin coadministration reduced trospium steady-state maximum plasma concentration (by $34 \%$ ) and area under the concentration-time curve from 0-24 hours (by $29 \%$ ). Neither
\end{abstract}

M. G. Oefelein · W. Tong $(\bowtie) \cdot$ S. Kerr · K. Bhasi ·

R. K. Patel · D. Yu

Allergan, Inc., 2525 Dupont Drive, Irvine, CA 92612, USA

e-mail: tong_warren@allergan.com drug's renal clearance was affected. No safety/tolerability issues of concern were observed with coadministration. Conclusion No dosage adjustment is necessary for metformin when coadministered with trospium chloride XR.

\section{Introduction}

Overactive bladder $(\mathrm{OAB})$ is a common condition, affecting an estimated $17 \%-19 \%$ of adults in the USA [1,2]. $\mathrm{OAB}$ is associated with a number of co-morbid medical conditions, including diabetes mellitus and obesity [3-6]. Co-morbid conditions may necessitate concurrent treatment with any $\mathrm{OAB}$ therapies, allowing for the potential for drug-drug interactions (DDIs).

The quaternary amine antimuscarinic trospium differs from the tertiary amine antimuscarinics used to treat $\mathrm{OAB}$ in that it is renally eliminated rather than metabolized by the cytochrome P450 (CYP450) pathway; therefore, trospium has a low propensity for DDIs with drugs metabolized by CYP450 [7]. Of the dose absorbed $(<10 \%)$ following oral administration, approximately $60 \%-80 \%$ of the parent compound is excreted unchanged in the urine [7-9]. Trospium has been hypothesized to undergo ester hydrolysis with subsequent conjugation of benzylic acid to form azoniaspironortropanol with glucuronic acid, which is known to account for only approximately $7 \%-10 \%$ of urinary excretion. The mean renal clearance for trospium is approximately four times higher than the average glomerular filtration rate, indicating that active tubular secretion is a major route of elimination for trospium [7, 8]. Therefore, concomitant administration of trospium chloride with another renally excreted drug, particularly those that also undergo tubular secretion, may result in a DDI if they compete for the same transporter, e.g., organic cation 
transporters (OCTs). However, a pharmacokinetic study indicated no DDI between trospium chloride and digoxin, which is renally eliminated [10].

The biguanide oral antihyperglycaemic agent metformin is indicated for the treatment of patients with type 2 diabetes mellitus, in whom use of the drug is associated with improved glycaemic control and stable or slightly decreased body weight [11]. Metformin may also be used to reduce progression to type 2 diabetes in patients at risk [12-15], and has been shown to promote weight loss in patients with obesity [16-19]. Metformin was the sixth most commonly prescribed medication in the USA in 2009 [20], and it is the most widely prescribed oral medication for the treatment of diabetes [21]. Metformin is primarily excreted unchanged in the urine, without hepatic metabolism or biliary secretion [11]. The renal clearance of metformin is approximately 3.5 times greater than creatinine clearance, indicating that tubular secretion is a major route of elimination for the drug [7]. Approximately $90 \%$ of the absorbed metformin dose is eliminated renally within the first 24 hours following oral administration [11]. No metabolites have been identified following administration of metformin to humans $[11,22]$.

Given that $\mathrm{OAB}$ is a common co-morbidity of diabetes, metformin and trospium chloride are likely to be co-prescribed and, since both drugs undergo renal excretion, there is a potential for each drug to affect the systemic exposure and/or half-life of the other drug. Human OCTs (hOCTs) are involved in both the intestinal absorption and renal and/ or hepatic elimination of metformin $\left(\mathrm{hOCT}_{1,2}\right)$ and trospium ( $\mathrm{hOCT}_{1,2,3}$ ), and could contribute to their active tubular and hepatobiliary secretion [23, 24]. Consequently, it is possible that transport of one or both of these agents might be inhibited by the administration of the other agent, thus affecting their absorption and/or elimination. Therefore, the purpose of this study was to investigate the pharmacokinetics, safety and tolerability of coadministration of trospium chloride extended release (XR) with metformin under steady-state conditions.

\section{Methods}

This single-centre, randomized, open-label, two-period, crossover study (Fig. 1) enrolled healthy male and female subjects aged 18-45 years weighing within $\pm 30 \%$ of their ideal body weight for height and frame size (according to Metropolitan height and weight tables). Subjects were required to be non-smokers (refraining from tobacco usage, including smokeless tobacco, nicotine patches, etc., for 1 month prior to screening visit). In addition, subjects had to abstain from caffeine-containing food and beverages for 24 hours prior to dosing, alcohol-containing food and beverages for 48 hours prior to dosing, and Seville oranges (e.g., orange marmalade), grapefruit or grapefruit juice for 7 days prior to dosing, and from all such products throughout the study.

This crossover study was an $\mathrm{AB} \rightarrow \mathrm{BA}$ sequence design with all subjects completing two crossover periods (Fig. 1). Due to the long half-life of trospium chloride XR (consequently requiring a long drug washout period), the sequence effect of trospium chloride XR could not be determined because the combination treatment is always administered after the trospium chloride XR alone treatment. The protocol was designed so that the washout time for the long-acting trospium chloride XR formulation was kept to a reasonable length of time while not appreciably altering the interpretation of the DDI study. The study design was consistent with US Food and Drug Administration guidance for long half-life DDI studies.

Subjects received oral metformin (Glucophage ${ }^{\circledR}$; Bristol-Myers Squibb, Princeton, NJ, USA) or oral trospium chloride XR (Sanctura $\mathrm{XR}^{\circledR}$; Allergan, Inc., Irvine, CA, USA) according to the study protocol (Fig. 1). Subjects were instructed to take trospium chloride XR once daily at $7 \mathrm{am}$, on an empty stomach 1 hour before breakfast, and metformin twice daily at 8 am and $6 \mathrm{pm}$ with a standardized meal (2,500 calories/day with $30 \%$ fat). Overnight fasting was required before the scheduled clinic attendance on Days 4, 17 and 21 for Group A, and Days 10, 14 and 27 for Group B. The only concomitant medications permitted during the study were hormonal contraceptives and paracetamol (acetaminophen) $\leq 2 \mathrm{~g} /$ day .

\subsection{Pharmacokinetic Evaluation}

For the measurement of plasma and urine concentrations of trospium and metformin, serial blood and urine samples were collected from each subject. Collection time points for the metformin-alone treatment were pre-dose and 0.5 , $0.75,1,2,3,4,6,8,10,12,14,16$ and 24 h post-dose for plasma samples, and pre-dose and 0-4, 4-12 and 12-24 h post-dose for pooled urine samples on Day 4 for Group A and Day 27 for Group B. Collection time points for the trospium chloride XR-alone treatment were pre-dose and $1,2,3,4,5,6,8,10,12,16$ and $24 \mathrm{~h}$ post-dose for plasma samples, and pre-dose and $0-4,4-12$ and $12-24 \mathrm{~h}$ postdose for pooled urine samples on Day 10 for Group B and Day 17 for Group A. Collection time points following coadministration of trospium chloride XR and metformin were pre-trospium chloride XR dose and 1, 2, 3, 4, 5, 6, 8, $10,12,16,24,48,72,96,120$ and $144 \mathrm{~h}$ post-trospium chloride XR dose for plasma samples, and pre-metformin dose and $0-4,4-12$ and 12-24 h post-metformin dose for pooled urine samples on Day 14 for Group B and Day 21 


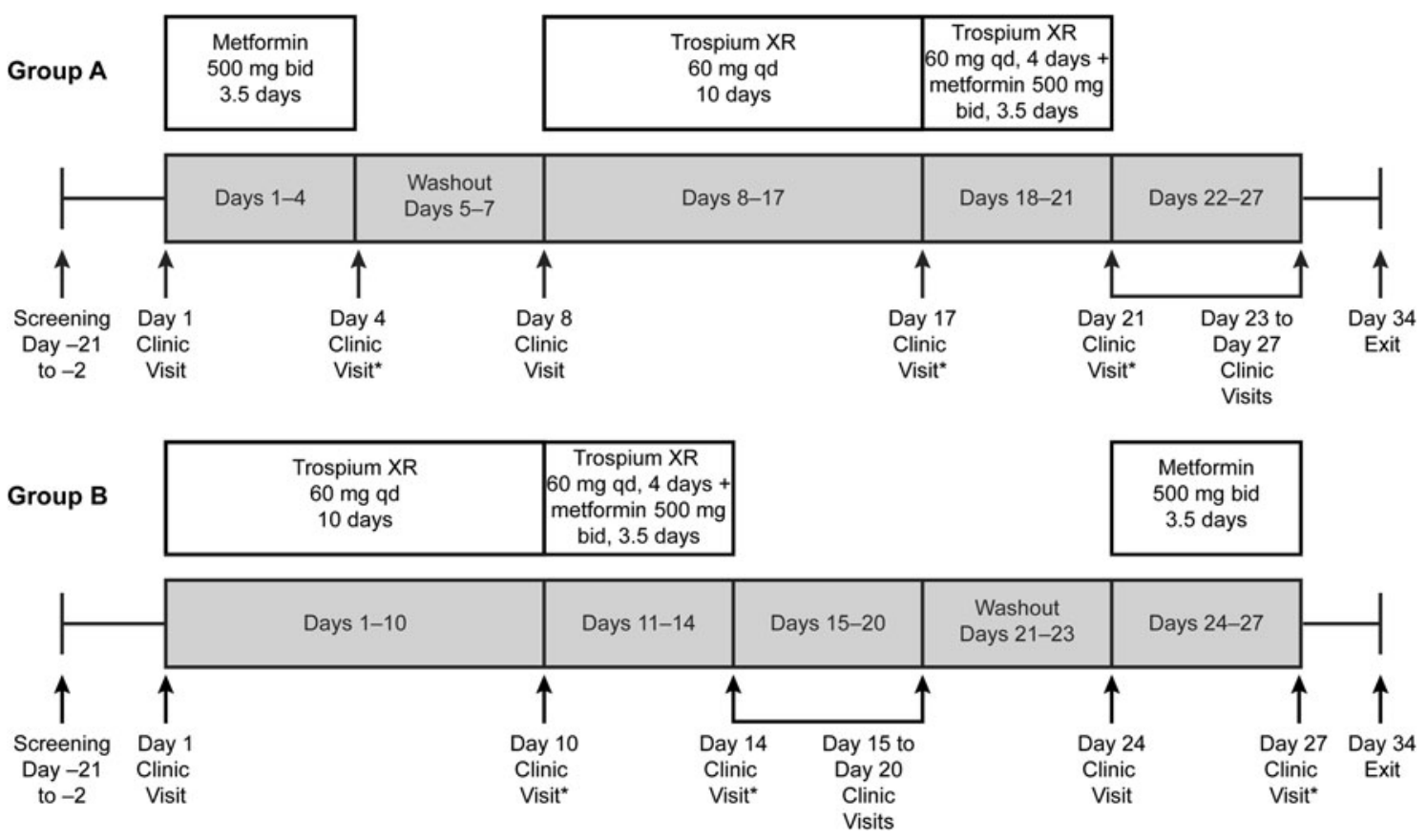

Fig. 1 Study design. Asterisks denote visits involving an overnight stay at clinic. bid twice daily, $q d$ once daily, $X R$ extended release

for Group A. The final plasma pharmacokinetic sample was collected on Day 34 at $312 \mathrm{~h}$ post-dose for Group A, and at $168 \mathrm{~h}$ post-dose for Group B.

Plasma and urine samples were analysed at Prevalere Life Sciences, LLC (Whitesboro, NY, USA). Plasma and urine trospium concentrations were measured using a fully validated liquid chromatography-tandem mass spectrometry (LC-MS/MS) method with a lower limit of quantitation (LLQ) of $0.04 \mathrm{ng} / \mathrm{mL}$ and $25 \mathrm{ng} / \mathrm{mL}$, respectively. Plasma and urine metformin concentrations were measured using a validated LC-MS/MS method with an LLQ of $10 \mathrm{ng} / \mathrm{mL}$ and $1 \mu \mathrm{g} / \mathrm{mL}$, respectively.

\subsection{Safety and Tolerability Evaluation}

Adverse events (AEs) were recorded throughout the study period. Clinical laboratory evaluations (haematology, biochemistry and urinalysis) were performed at screening and at the final day of the two treatment periods (Day 21 for Group A and Day 14 for Group B). Vital signs (heart rate, blood pressure, respiration rate and oral body temperature) were measured and 12-lead standard electrocardiograms (ECGs) performed at screening, the final day of the two treatment periods (Day 21 for Group A and Day 14 for Group B), and at the final study exit (Day 35). Physical examinations were performed at screening, Day 8 (Group A) and Day 24 (Group B), and study exit (Day 35). Body weight was measured at screening and study exit (Day 35).

\subsection{Statistical Methods}

\subsubsection{Sample Size Calculation}

The sample size required to power the study of steady-state bioequivalence between trospium chloride XR and metformin was driven by within-subject variability as measured by the coefficient of variation for trospium chloride XR $(40 \%)$. With a sample size of 18 in each sequence (total of 36 subjects), a crossover design would yield $80 \%$ power to reject both the null hypothesis that the ratio of the test mean to the standard mean is $<0.80$ and the null hypothesis that the ratio of the test mean to the standard mean is $>1.25$ (i.e., the test and the standard are not bioequivalent), in favour of the alternative hypothesis that the means of the two treatments are bioequivalent. Accounting for a $15 \%$ drop-out rate, it was anticipated that approximately 44 subjects would need to be enrolled into the study in order to have 36 subjects complete the study.

\subsubsection{Pharmacokinetic Analyses}

The calculation of pharmacokinetic parameters was performed using WinNonlin ${ }^{\circledR}$ Enterprise Version 5.2 with IVIVC Toolkit (Pharsight Corporation, Mountain View, CA, USA) and SAS $^{\circledR}$ for Windows Version 9.1 (SAS Institute, Inc., Cary, NC, USA). The summary statistics of the pharmacokinetic parameters were calculated using SAS $^{\circledR}$. SAS ${ }^{\circledR}$ was also used for data management and other 
statistical analyses. S-Plus ${ }^{\circledR} 7.0$ (TIBCO Software, Inc., Seattle, WA, USA, formerly Insightful Corporation) was used for the graphing of pharmacokinetic data.

Plasma: Trospium and Metformin A model-independent approach was used to calculate the plasma and urine pharmacokinetic parameters of trospium and metformin for each subject. Plasma pharmacokinetic parameters, including steady-state area under the plasma concentration-time curve (AUC) within the dosing interval (AUC from time zero to 12 hours $\left[\mathrm{AUC}_{12}\right.$ ] for metformin and AUC from time zero to 24 hours $\left[\mathrm{AUC}_{24}\right.$ ] for trospium), maximum (peak) drug concentration $\left(\mathrm{C}_{\max }\right)$, time to peak plasma concentration $\left(\mathrm{t}_{\max }\right)$ following drug administration, oral clearance and half-life, were calculated when applicable. Descriptive statistics were determined for all pharmacokinetic parameters calculated.

Pharmacokinetic DDIs were evaluated by calculating the $90 \%$ confidence interval (CI) of the ratio of estimated geometric means for plasma $\mathrm{AUC}_{12}$ (metformin), $\mathrm{AUC}_{24}$ (trospium) and $\mathrm{C}_{\max }$ values obtained following administration, alone and in combination. Administration alone was used as the reference for each respective drug.

Urine: Trospium and Metformin The cumulative amount of unchanged drug excreted in the urine $\left(\mathrm{A}_{\mathrm{e}}\right)$ during the 12or 24-h dosing interval $\left(A_{e}\right.$ from time zero to 12 hours for metformin and $\mathrm{A}_{\mathrm{e}}$ from time zero to 24 hours for trospium) was calculated for each subject. The percentage of dose excreted in urine was determined from the ratio of $A_{e}$ to the administered dose. Renal clearance of drug from plasma $\left(\mathrm{CL}_{\mathrm{R}}\right)$ was determined by dividing $\mathrm{A}_{\mathrm{e}}$ by the plasma AUC of the corresponding dosing interval of each drug. A paired Student's $t$ test was performed to compare these parameters between administration alone and in combination for each compound at the significance level of 0.05 .

\subsubsection{Safety Analyses}

All randomized subjects who received at least one dose of study medication were included in the safety analyses. AE data were summarized by frequency tables. Summary statistics were calculated with $\mathrm{SAS}^{\circledR}$ for the change from baseline in laboratory parameters, ECG parameters and vital signs.

\subsubsection{Post hoc Efficacy Analysis}

To determine the clinical implications of the reduced trospium exposure during concomitant metformin administration, a post hoc analysis was performed on efficacy data reported from two multicentre, randomized, doubleblind, placebo-controlled Phase III trials [25, 26].
Comparison of primary efficacy outcomes (average number of toilet voids and urinary urge incontinence [UUI] episodes per day) was conducted between subjects who received both metformin and trospium chloride XR, and an equal number of subjects not on metformin but who received trospium chloride XR only (i.e., 1:1 matching). Sensitivity analyses were also performed using twice the number of control subjects (i.e., 1:2 matching). Additionally, primary efficacy outcomes were compared between subjects on metformin and all subjects who did not receive metformin treatment (without matching). Control subjects were selected by propensity score matching [27], with propensity scores estimated using logistic regression with metformin (yes vs. no) as a dependent variable, and age, sex, race, OAB duration (years), baseline urinary frequency, baseline urgency severity and baseline UUI episodes per day as independent variables.

\subsection{Ethics}

The study was conducted in accordance with the applicable Good Clinical Practice regulations and guidelines and Institutional Review Board (IRB) regulations. The study was approved by Aspire IRB, La Mesa, CA, USA on 16 September 2009. All subjects provided written informed consent prior to enrollment.

\section{Results}

A total of 44 subjects ( 25 males and 19 females) were randomized to receive study treatment. Of these, 43 subjects completed the study and one subject in Group B discontinued at Day 13 due to flu-like symptoms considered unrelated to the study drug during the H1N1 pandemic of 2009. The mean age was 31 years (range 18-44 years), mean height was $165 \mathrm{~cm}$ (range 153-180 cm), and mean body weight was $69.5 \mathrm{~kg}$ (range $55.3-87.9 \mathrm{~kg}$ ). All 44 subjects were Caucasian.

\subsection{Pharmacokinetics}

\subsubsection{Metformin}

The steady-state plasma concentration-time profiles of metformin following administration of metformin alone and in combination with trospium chloride XR are shown in Figure 2. Coadministration of trospium chloride XR did not alter the steady-state plasma and urine pharmacokinetics of metformin (Table 1). Based on the metformin steady-state $\mathrm{C}_{\max }$ and $\mathrm{AUC}_{12}$ values, administration of metformin alone and in combination with trospium chloride XR were bioequivalent; the $90 \%$ CIs for the ratios of 


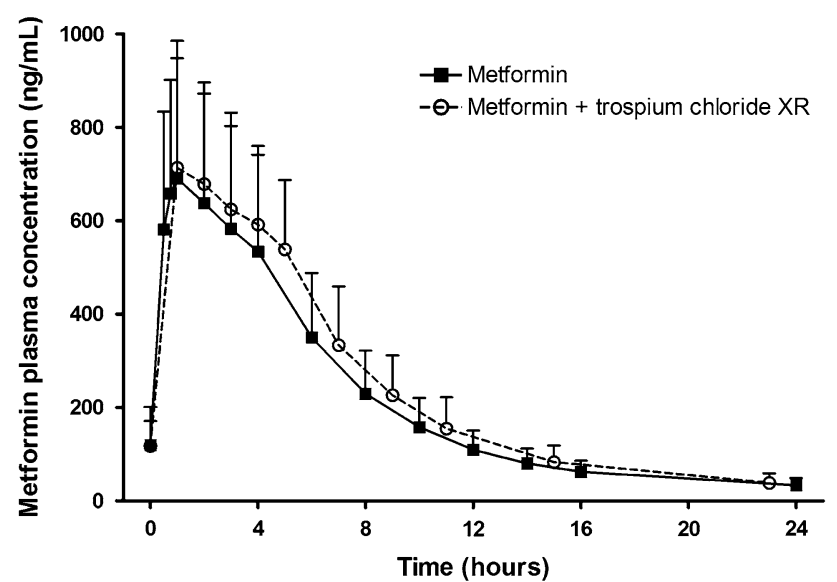

Fig. 2 Steady-state plasma concentration-time profiles of metformin following oral administration of metformin $500 \mathrm{mg}$ twice daily alone and in combination with trospium chloride XR $60 \mathrm{mg}$ once daily (mean \pm standard deviation, $n=43$ ). $X R$ extended release

Table 1 Steady-state plasma and urine pharmacokinetic parameters of metformin following oral administration of metformin $500 \mathrm{mg}$ twice daily, alone and in combination with trospium chloride XR $60 \mathrm{mg}$ once daily (mean \pm standard deviation)

\begin{tabular}{|c|c|c|}
\hline $\begin{array}{l}\text { Pharmacokinetic } \\
\text { parameters of } \\
\text { metformin }\end{array}$ & $\begin{array}{l}\text { Metformin } \\
(n=43)\end{array}$ & $\begin{array}{l}\text { Metformin }+ \text { trospium } \\
\text { chloride XR }(n=43)\end{array}$ \\
\hline $\mathrm{C}_{\max }(\mathrm{ng} / \mathrm{mL})$ & $739 \pm 263$ & $753 \pm 252$ \\
\hline $\mathrm{t}_{\max }(\mathrm{h})$ & $1.39 \pm 1.04$ & $1.82 \pm 1.10$ \\
\hline $\mathrm{AUC}_{12}(\mathrm{ng} \cdot \mathrm{h} / \mathrm{mL})$ & $4,450 \pm 1,590$ & $4,900 \pm 1,550$ \\
\hline$t_{1 / 2}(h)$ & $7.14 \pm 3.37$ & $5.73 \pm 1.62$ \\
\hline $\mathrm{CL}_{\mathrm{ss}} / \mathrm{F}(\mathrm{L} / \mathrm{h})$ & $127 \pm 46$ & $110 \pm 27$ \\
\hline $\mathrm{A}_{\mathrm{e}, 12}(\mathrm{mg})$ & $141 \pm 50$ & $160 \pm 44$ \\
\hline $\mathrm{A}_{\mathrm{e}, 12} /$ dose $(\%)$ & $28.3 \pm 10.0$ & $31.9 \pm 8.8$ \\
\hline $\mathrm{CL}_{\mathrm{R}}(\mathrm{L} / \mathrm{h})$ & $33.0 \pm 8.5$ & $33.7 \pm 8.5$ \\
\hline
\end{tabular}

$A_{e, 12}$ cumulative amount of drug excreted in the urine from time zero to 12 hours, $A U C_{12}$ area under the plasma concentration-time curve from time zero to 12 hours, $C I$ confidence interval, $C L_{R}$ renal clearance of drug from plasma, $C L_{S S} / F$ steady-state apparent body clearance of drug from plasma after oral administration, $C_{\max }$ maximum (peak) plasma drug concentration, $t_{1 / 2}$ terminal elimination half-life, $t_{\max }$ time to reach maximum (peak) plasma concentration following drug administration, $X R$ extended release

combination/alone were 97-109 (percentage ratio of the geometric mean $=103$ ) for $\mathrm{C}_{\max }$ and 105-120 (percentage ratio of the geometric mean $=112$ ) for $\mathrm{AUC}_{12}$ (Table 1).

Following administration of metformin alone and in combination with trospium chloride $\mathrm{XR}$, comparable mean values were observed for the $\mathrm{CL}_{\mathrm{R}}$ of metformin (33.0 and $33.7 \mathrm{~L} / \mathrm{h}$, respectively; $p=0.41$ ) and the percentage of dose excreted in urine ( $28.3 \%$ and $31.9 \%$, respectively), although the increase of approximately $13 \%$ in percentage of dose excreted in urine was statistically significant $(p<0.05$; Table 1).

\subsubsection{Trospium Chloride Extended Release}

The steady-state plasma concentration-time profiles of trospium following administration of trospium chloride XR alone and in combination with metformin are shown in Figure 3. Coadministration of metformin did not alter the absorption rate of trospium from trospium chloride XR, with $t_{\max }$ being approximately 4 hours for trospium chloride $\mathrm{XR}$, both alone and in combination with metformin (Table 2).

Administration of trospium chloride XR with metformin, compared with trospium chloride $\mathrm{XR}$ alone, reduced the trospium steady-state $\mathrm{C}_{\max }$ and $\mathrm{AUC}_{24}$ values by approximately $34 \%$ and $29 \%$, respectively (Table 2). Although the percentage of trospium chloride XR dose excreted in urine was reduced by $30 \%(p<0.0001)$, there was no statistically significant change in the $C_{R}$ of trospium when the drugs were coadministered $(p=0.88$; Table 2). Based on the trospium steady-state $C_{\max }$ and $\mathrm{AUC}_{24}$ values, administration of trospium chloride $\mathrm{XR}$ alone and in combination with metformin resulted in $90 \%$ CIs for the ratios of geometric mean (combination/alone) of $61-72$ (percentage ratio of the geometric mean $=66.3$ ) for $\mathrm{C}_{\max }$ and 66-76 (percentage ratio of the geometric mean $=70.8)$ for $\mathrm{AUC}_{24}$ (Table 2).

\subsection{Safety and Tolerability}

There were no serious AEs reported during this study, and no subject discontinued the study due to an AE. A total of 97 treatment-emergent AEs (TEAEs) were reported by 26 subjects $(59 \%)$; these occurred in 11 subjects $(26 \% ; 43$ TEAEs) following administration of metformin alone, eight subjects (18\%; 15 TEAEs) following administration

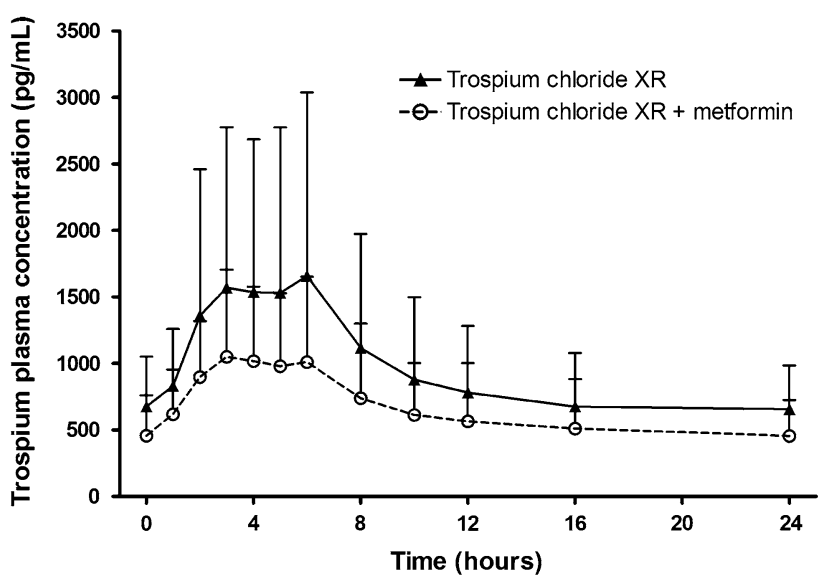

Fig. 3 Steady-state plasma concentration-time profiles of trospium following oral administration of trospium chloride XR $60 \mathrm{mg}$ once daily alone and in combination with metformin $500 \mathrm{mg}$ twice daily (mean \pm standard deviation, $n=43$ ). $X R$ extended release 
Table 2 Steady-state plasma and urine pharmacokinetic parameters of trospium following oral administration of trospium chloride XR $60 \mathrm{mg}$ once daily, alone and in combination with metformin $500 \mathrm{mg}$ twice daily (mean \pm standard deviation)

\begin{tabular}{lcc}
\hline $\begin{array}{l}\text { Pharmacokinetic } \\
\text { parameters } \\
\text { of trospium } \\
\text { chloride XR }\end{array}$ & $\begin{array}{l}\text { Trospium } \\
\text { chloride XR } \\
(n=44)\end{array}$ & $\begin{array}{l}\text { Trospium chloride } \\
\text { XR }+ \text { metformin } \\
(n=43)\end{array}$ \\
\hline $\mathrm{C}_{\max }(\mathrm{ng} / \mathrm{mL})$ & $1.87 \pm 1.44$ & $1.17 \pm 0.71$ \\
$\mathrm{t}_{\max }(\mathrm{h})$ & $4.42 \pm 1.94$ & $4.12 \pm 1.60$ \\
$\mathrm{AUC}_{24}(\mathrm{ng} \cdot \mathrm{h} / \mathrm{mL})$ & $22.7 \pm 14.4$ & $15.6 \pm 9.5$ \\
$\mathrm{CL}_{\mathrm{ss}} / \mathrm{F}(\mathrm{L} / \mathrm{h})$ & $3,510 \pm 1,920$ & $4,830 \pm 2,320$ \\
$\mathrm{~A}_{\mathrm{e}, 24}(\mathrm{mg})$ & $0.400 \pm 0.247$ & $0.281 \pm 0.156$ \\
$\mathrm{~A}_{\mathrm{e}, 24} / \mathrm{dose}(\%)$ & $0.667 \pm 0.411$ & $0.468 \pm 0.260$ \\
$\mathrm{CL}_{\mathrm{R}}(\mathrm{L} / \mathrm{h})$ & $18.4 \pm 4.8$ & $18.4 \pm 5.4$ \\
\hline
\end{tabular}

$A_{e, 24}$ cumulative amount of drug excreted in the urine from time zero to 24 hours, $A U C_{24}$ area under the plasma concentration-time curve from time zero to 24 hours, $C I$ confidence interval, $C L_{R}$ renal clearance of drug from plasma, $C L_{S S} / F$ steady-state apparent body clearance of drug from plasma after oral administration, $C_{\max }$ maximum (peak) plasma drug concentration, $t_{\max }$ time to reach maximum (peak) plasma concentration following drug administration, $X R$ extended release

of trospium chloride XR alone, and 17 subjects (39\%; 39 TEAEs) following administration of trospium chloride XR in combination with metformin. The most common TEAEs were headache and diarrhoea (Table 3). Of the 97 TEAEs, 95 were mild in severity and two were moderate (headache).

Mean laboratory, vital sign and ECG parameters remained within reference range at all assessed post-dose time points and varied minimally from baseline. At baseline, the mean heart rate was 69 beats/min (range 45-92 beats/min). On Day 1 of treatment, the mean change in heart rate from baseline in Group A (metformin) and Group B (trospium chloride XR + metformin) was +4.9 and +3.4 beats $/ \mathrm{min}$, respectively, when measured $2 \mathrm{~h}$ postdose. In Group B, the mean change in heart rate from baseline on Day 11 was +6.1 beats/min when measured immediately before dosing (pre-dose), and +13.1 beats/ min when measured $2 \mathrm{~h}$ post-dose. Similarly, on Day 14 the mean pre-dose and post-dose changes in heart rate in Group B were +5.1 and +8.8 beats/min, respectively.

There were no post-dose abnormal physical examination findings. Mean weight from baseline to study exit increased by $0.07 \mathrm{~kg}$ for Group A and $0.56 \mathrm{~kg}$ for Group B.

\subsection{Post hoc Efficacy Analysis}

Post hoc efficacy analyses from the pivotal Phase III studies revealed that no statistical difference on the primary efficacy outcomes at Week 12 (average number of toilet voids/day or average number of UUI episodes/day) was observed between patients on concomitant metformin and trospium chloride XR therapy, and propensity score-matched trospium chloride XR patients who were not on metformin therapy (Table 4).

\section{Discussion}

The primary finding of this DDI study is that the steadystate pharmacokinetics of metformin were not affected by
Table 3 Treatment-emergent adverse events occurring in $\geq 5 \%$ of subjects following oral administration of metformin alone, trospium chloride XR alone, or metformin and trospium chloride XR in combination [n (\%)]

$X R$ extended release

\begin{tabular}{lllll}
\hline Adverse event & $\begin{array}{l}\text { Metformin } \\
(n=43)\end{array}$ & $\begin{array}{l}\text { Trospium } \\
\text { chloride XR } \\
(n=44)\end{array}$ & $\begin{array}{l}\text { Trospium chloride } \\
\text { XR }+ \text { metformin } \\
(n=44)\end{array}$ & $\begin{array}{l}\text { Total } \\
(N=44)\end{array}$ \\
\hline Headache & $4(9)$ & $3(7)$ & $7(16)$ & $12(27)$ \\
Diarrhoea & $3(7)$ & $0(0)$ & $3(7)$ & $6(14)$ \\
Abdominal distension & $1(2)$ & $2(5)$ & $0(0)$ & $3(7)$ \\
Abdominal pain, upper & $3(7)$ & $0(0)$ & $0(0)$ & $3(7)$ \\
Constipation & $1(2)$ & $1(2)$ & $1(2)$ & $3(7)$ \\
Dizziness & $1(2)$ & $1(2)$ & $1(2)$ & $3(7)$ \\
Dry throat & $1(2)$ & $2(5)$ & $0(0)$ & $3(7)$ \\
Nasal congestion & $0(0)$ & $0(0)$ & $3(7)$ & $3(7)$ \\
Abdominal pain & $0(0)$ & $1(2)$ & $2(5)$ & $2(5)$ \\
Dry lip & $2(5)$ & $0(0)$ & $0(0)$ & $2(5)$ \\
Metrorrhagia & $2(5)$ & $0(0)$ & $0(0)$ & $2(5)$ \\
Nausea & $2(5)$ & $0(0)$ & $1(2)$ & $2(5)$ \\
Oropharyngeal pain & $0(0)$ & $1(2)$ & $1(2)$ & $2(5)$ \\
Upper respiratory tract infection & $0(0)$ & $0(0)$ & $2(5)$ & $2(5)$ \\
Vomiting & $1(2)$ & $0(0)$ & $1(2)$ & $2(5)$ \\
White blood cells urine positive & $1(2)$ & $1(2)$ & $0(0)$ & \\
\hline
\end{tabular}


Table 4 Propensity score matching results: change from baseline at Week 12 in toilet voids and UUI episodes in subjects administered trospium chloride XR with and without metformin [N or mean (SD)]

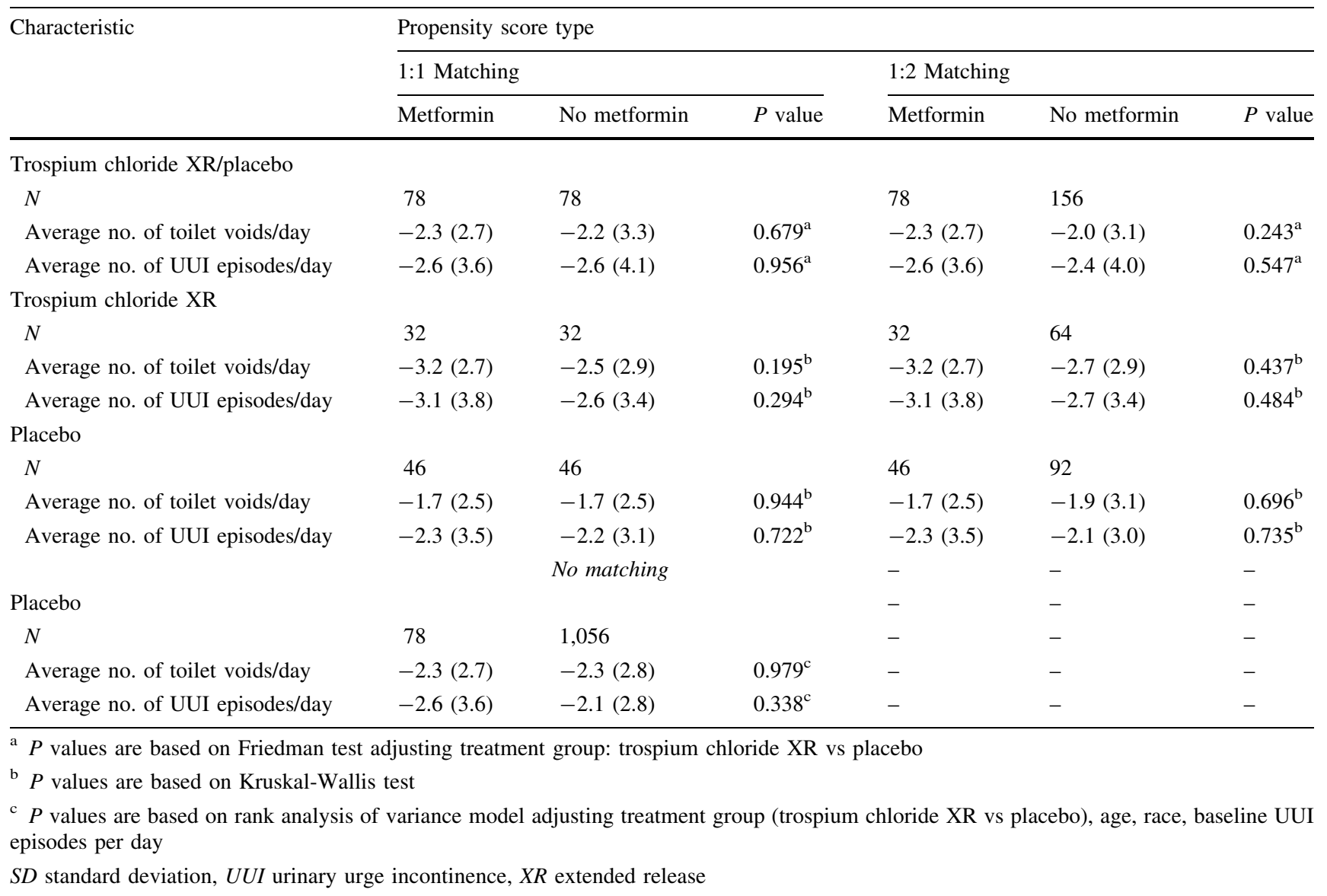

concomitant administration of trospium chloride XR in healthy subjects. Notably, the $\mathrm{CL}_{\mathrm{R}}$ of metformin and trospium was not changed significantly by coadministration compared with administration of either drug alone.

It is important to evaluate whether any pharmacokinetic DDI occurs between trospium and metformin, both of which are eliminated via renal tubular secretion, as there is considerable potential for these drugs to be concurrently prescribed to patients with OAB. Metformin was the sixth most commonly prescribed medication in the USA in 2009 [20], and it is the most widely prescribed oral treatment for diabetes [21]. Metformin is indicated for the treatment of type 2 diabetes, and may be used to prevent progression to type 2 diabetes and to treat obesity. Diabetes (with type 2 diabetes accounting for $90 \%-95 \%$ of diabetes cases in adults) [28] and obesity are highly prevalent in the USA and worldwide; [29-32] furthermore, diabetes and obesity are often co-morbid with $\mathrm{OAB}$ [3-6]. Among the pooled population of patients enrolled in Phase III trospium chloride XR studies, the mean body mass index (BMI) was $32 \mathrm{~kg} / \mathrm{m}^{2}$, which exceeds the criterion used to define obesity in adults $\left(\geq 30 \mathrm{~kg} / \mathrm{m}^{2}\right)$ [33]. A subanalysis of data from these pivotal studies showed that the severity of $\mathrm{OAB}$ increased with BMI; however, trospium chloride XR was similarly efficacious, and significantly more effective than placebo, across all BMI strata [33].

Trospium is primarily eliminated unchanged via renal filtration and tubular secretion, rather than being metabolized by CYP450. A theoretical DDI exists between trospium and other renally eliminated drugs, such as metformin [7], if there is competition between the agents for the same transporter. Of note, a previous DDI study showed that trospium chloride did not alter the pharmacokinetics of digoxin, which is primarily eliminated by renal excretion [10]. The current study expands on these findings, demonstrating that trospium does not alter the pharmacokinetics of metformin.

An interesting secondary finding of the present study was the observation that coadministration of trospium chloride XR with metformin resulted in reductions in the mean steady-state $\mathrm{C}_{\max }(34 \%)$ and $\mathrm{AUC}_{24}(29 \%)$ of trospium. On the surface, this observation suggests that coadministration of metformin with trospium chloride XR has the potential to interfere with the therapeutic efficacy of the latter agent. However, this and other studies have shown that there is very wide interindividual variability in 
trospium plasma levels after administration of trospium chloride XR. For example, the mean $\mathrm{AUC}_{24}$ of trospium was $20.2 \pm 14.2 \mathrm{ng} \cdot \mathrm{h} / \mathrm{mL}$ on Day 10 of administration in another study of this formulation in healthy adult subjects, which is intermediate between the mean values obtained with and without concomitant metformin administration in the present study [34]. Moreover, Silver et al. [34] observed moderate to high (35\%-90\%) intersubject variability in their study, and estimated the peak-to-trough ratio $\left(\mathrm{C}_{\max } / \mathrm{C}_{\min }\right)$ to range from 2 to 13 . The trospium chloride XR formulation produced a lower $\mathrm{C}_{\max }$ than that of the immediate-release (IR) formulation in a head-tohead comparison in healthy subjects [34]. This marked change in pharmacokinetics resulted in no significant change in the efficacy of the XR formulation relative to the IR formulation, but was associated with improved tolerability. Finally, as demonstrated in the above post hoc analysis of data from the Phase III trials, the efficacy of trospium chloride XR was not affected by metformin coadministration, suggesting that the lower exposure of trospium exposure during metformin coadministration is not clinically significant.

As noted above, a subanalysis of data from Phase III trials of trospium chloride XR showed that the efficacy of the drug was consistent across BMI strata. Although the study by Chancellor et al. [33] did not evaluate the impact of metformin coadministration on the efficacy of trospium chloride XR, the finding that efficacy was not impaired in obese patients, who would be expected to be more likely to be receiving antidiabetic agents, is reassuring.

Both metformin and trospium are substrates of the proximal gut cation transporter system [23, 24]. Our hypothesis is that metformin has a higher affinity/efficiency of absorption compared with trospium chloride and, therefore, competition between metformin and trospium for the transporter limits absorption of trospium from the intestine when the two drugs are coadministered. Apparently, this competition did not take place in the renal secretion process, as the renal clearance of trospium was not affected. This might explain the observation in the current study that trospium plasma concentration decreases while clearance and dosing are unchanged. This hypothesis will need to be specifically tested and confirmed via an in vitro cellular culture model in which the OCT system is expressed.

A potential limitation of the current study, as is typical of pharmacokinetic studies, is that it was conducted in healthy subjects with normal renal function; therefore, the results observed in the study subjects may not be representative of those that would be observed in patients with co-morbid medical conditions that would necessitate concomitant prescribing of these medications. However, it should be noted that because both drugs are eliminated renally, neither metformin nor trospium chloride $\mathrm{XR}$ are recommended for use in patients with severe renal impairment (trospium chloride XR is not recommended for use in patients with a creatinine clearance $<30 \mathrm{~mL} / \mathrm{min}$; metformin is contraindicated in patients with a serum creatinine concentration $\geq 1.5 \mathrm{mg} / \mathrm{dL}$ [men] or $\geq 1.4 \mathrm{mg} / \mathrm{dL}$ [women] or an abnormal creatinine clearance) [7, 11].

\section{Conclusion}

Coadministration of trospium chloride XR $60 \mathrm{mg}$ once daily and metformin $500 \mathrm{mg}$ twice daily did not affect the steady-state pharmacokinetics of metformin. Therefore, no dosage adjustment is necessary for metformin when it is coadministered with trospium chloride XR. Coadministration of metformin reduced the steady-state systemic exposure of trospium; however, renal clearance of trospium remained unchanged. As this study was conducted in healthy adult subjects, the results may not be generalizable to patients with $\mathrm{OAB}$ in a clinical setting.

Acknowledgements This study was funded by Allergan, Inc.

Alan J. Klopp, PhD, Sushma Soni and Mary Hines of inScience Communications, Springer Healthcare, provided editorial support, which was funded by Allergan, Inc.

Financial disclosures: Warren Tong, PharmD, MS, Sam Kerr, $\mathrm{PhD}$, Kavitha Bhasi, PhD, and Rina K. Patel, MS, are all employees of Allergan, Inc. Dale Yu, PhD, and Michael G. Oefelein, MD, were employees of Allergan, Inc. at the time of the study.

Open Access This article is distributed under the terms of the Creative Commons Attribution Noncommercial License which permits any noncommercial use, distribution, and reproduction in any medium, provided the original author(s) and the source are credited.

\section{References}

1. Stewart WF, Van Rooyen JB, Cundiff GW, et al. Prevalence and burden of overactive bladder in the United States. World J Urol. 2003;20(6):327-36.

2. Onukwugha E, Zuckerman IH, McNally D, et al. The total economic burden of overactive bladder in the United States: a disease-specific approach. Am J Manag Care. 2009;15(4 Suppl): S90-7.

3. McGrother CW, Donaldson MM, Hayward T, et al. Urinary storage symptoms and comorbidities: a prospective population cohort study in middle-aged and older women. Age Ageing. 2006;35(1):16-24.

4. Teleman PM, Lidfeldt J, Nerbrand C, et al. Overactive bladder: prevalence, risk factors and relation to stress incontinence in middle-aged women. BJOG. 2004;111(6):600-4.

5. Lawrence JM, Lukacz ES, Liu IL, et al. Pelvic floor disorders, diabetes, and obesity in women: findings from the Kaiser Permanente Continence Associated Risk Epidemiology Study. Diabetes Care. 2007;30(10):2536-41. 
6. Dallosso HM, McGrother CW, Matthews RJ, et al. The association of diet and other lifestyle factors with overactive bladder and stress incontinence: a longitudinal study in women. BJU Int. 2003;92(1):69-77.

7. Sanctura XR (trospium chloride extended release capsules). US prescribing information. Irvine: Allergan, Inc.; 2008.

8. Doroshyenko O, Jetter A, Odenthal KP, et al. Clinical pharmacokinetics of trospium chloride. Clin Pharmacokinet. 2005;44(7): 701-20.

9. Fusgen I, Hauri D. Trospium chloride: an effective option for medical treatment of bladder overactivity. Int J Clin Pharmacol Ther. 2000;38(5):223-34.

10. Sandage B, Sabounjian L, Shipley J, et al. Predictive power of an in vitro system to assess drug interactions of an antimuscarinic medication: a comparison of in vitro and in vivo drug-drug interaction studies of trospium chloride with digoxin. J Clin Pharmacol. 2006;46(7):776-84.

11. Glucophage (metformin hydrochloride) tablets; Glucophage XR (metformin hydrochloride) extended-release tablets. US prescribing information. Princeton: Bristol-Myers Squibb Company; 2009.

12. Paulweber B, Valensi P, Lindstrom J, et al. A European evidencebased guideline for the prevention of type 2 diabetes. Horm Metab Res. 2010;42(Suppl 1):S3-36.

13. American Diabetes Association. Standards of medical care in diabetes-2010. Diabetes Care. 2010;33(Suppl 1):S11-61.

14. Garber AJ, Handelsman Y, Einhorn D, et al. Diagnosis and management of prediabetes in the continuum of hyperglycemia: when do the risks of diabetes begin? A consensus statement from the American College of Endocrinology and the American Association of Clinical Endocrinologists. Endocr Pract. 2008;14(7):933-46.

15. Alberti KG, Zimmet P, Shaw J. International Diabetes Federation: a consensus on type 2 diabetes prevention. Diabet Med. 2007;24(5):451-63.

16. Lee A, Morley JE. Metformin decreases food consumption and induces weight loss in subjects with obesity with type II noninsulin-dependent diabetes. Obes Res. 1998;6(1):47-53.

17. Gokcel A, Gumurdulu Y, Karakose H, et al. Evaluation of the safety and efficacy of sibutramine, orlistat and metformin in the treatment of obesity. Diabetes Obes Metab. 2002;4(1):49-55.

18. Srinivasan S, Ambler GR, Baur LA, et al. Randomized, controlled trial of metformin for obesity and insulin resistance in children and adolescents: improvement in body composition and fasting insulin. J Clin Endocrinol Metab. 2006;91(6):2074-80.

19. Glueck CJ, Fontaine RN, Wang P, et al. Metformin reduces weight, centripetal obesity, insulin, leptin, and low-density lipoprotein cholesterol in nondiabetic, morbidly obese subjects with body mass index greater than 30. Metabolism. 2001;50(7):856-61.
20. Herper M. America's most popular drugs [online]. 2010; Available from URL: http://www.forbes.com/2010/05/11/narcoticpainkiller-vicodin-business-healthcare-popular-drugs.html. [Accessed September 3, 2011].

21. Holstein A, Stumvoll M. Contraindications can damage your health-is metformin a case in point? Diabetologia. 2005;48(12): 2454-9.

22. Scheen AJ. Clinical pharmacokinetics of metformin. Clin Pharmacokinet. 1996;30(5):359-71.

23. Wenge B, Geyer J, Bonisch $\mathrm{H}$. Oxybutynin and trospium are substrates of the human organic cation transporters. Naunyn Schmiedebergs Arch Pharmacol. 2011;383(2):203-8.

24. Zhou M, Xia L, Wang J. Metformin transport by a newly cloned proton-stimulated organic cation transporter (plasma membrane monoamine transporter) expressed in human intestine. Drug Metab Dispos. 2007;35(10):1956-62.

25. Staskin D, Sand P, Zinner N, et al. Once daily trospium chloride is effective and well tolerated for the treatment of overactive bladder: results from a multicenter phase III trial. J Urol. 2007;178(3 Pt 1):978-83; discussion 83-4.

26. Allergan, Inc. Data on file.

27. Coca-Perraillon M. Local and Global Optimal Propensity Score Matching, Paper 185-2007. Presented at the SAS Global Forum 2007, Orlando, Florida, USA; 2007; Paper 185-2007.

28. US Centers for Disease Control and Prevention. National diabetes fact sheet: general information and national estimates on diabetes in the United States, 2007 [online]. 2008; Available from URL: http://www.cdc.gov/diabetes/pubs/pdf/ndfs_2007.pdf [Accessed July 9, 2011].

29. Ogden CL, Carroll MD, Curtin LR, et al. Prevalence of overweight and obesity in the United States, 1999-2004. JAMA. 2006;295(13):1549-55.

30. Berghofer A, Pischon T, Reinhold T, et al. Obesity prevalence from a European perspective: a systematic review. BMC Public Health. 2008;8:200.

31. Mokdad AH, Bowman BA, Ford ES, et al. The continuing epidemics of obesity and diabetes in the United States. JAMA. 2001;286(10):1195-200.

32. Wild S, Roglic G, Green A, et al. Global prevalence of diabetes: estimates for the year 2000 and projections for 2030. Diabetes Care. 2004;27(5):1047-53.

33. Chancellor MB, Oefelein MG, Vasavada S. Obesity is associated with a more severe overactive bladder disease state that is effectively treated with once-daily administration of trospium chloride extended release. Neurourol Urodyn. 2010;29(4):551-4.

34. Silver N, Sandage B, Sabounjian L, et al. Pharmacokinetics of once-daily trospium chloride $60 \mathrm{mg}$ extended release and twicedaily trospium chloride $20 \mathrm{mg}$ in healthy adults. J Clin Pharmacol. 2010;50(2):143-50. 